\title{
Geometrical Constraints on the Cosmological Constant
}

\author{
M. D. Maia* \\ and G.S. Silva \\ Universidade de Brasília \\ Departamento de Matemática \\ 70.919 - Brasília, DF. Brasil \\ and \\ NASA/Fermilab Astrophysics Center \\ Fermi National Accelerator Laboratory \\ Box 500, Batavia, IL 60510-0500
}

July 27, 2021

\begin{abstract}
The cosmological constant problem is examined under the assumption that the extrinsic curvature of the space-time contributes to the vacuum. A compensation mechanism based on a variable cosmological term is proposed. Under a suitable hypothesis on the behavior of the extrinsic curvature, we find that an initially large $\Lambda(t)$ rolls down rapidly to zero during the early stages of the universe. Using perturbation analysis, it is shown that such vacuum behaves essentially as a spin-2 field which is independent of the metric.
\end{abstract}

*E-Mail: MARCOS@FNAlV.FNAL.GOV 


\section{Introduction}

Current astrophysical data based on the Friedmann-Robertson-Walker (FRW) cosmological model estimate an upper bound value for the effective cosmological constant, so that $\Lambda_{\text {eff }} / 8 \pi G$ is very small, to the order of $10^{-47} \mathrm{Gev}^{4}$. In order to match the contributions to the vacuum energy density arising from gravity coupled fields, of the order of $\left\langle\rho_{v}>\approx 10^{71} \mathrm{Gev}^{4}\right.$, the bare cosmological constant $\Lambda_{0}$ in Einstein's equations would have to cancel a very large difference of around 118 decimal places, requiring an endless fine tuning in a continuously expanding universe [1].

This fundamental problem could in principle be solved by the use of a simple and elegant generalization of Einstein's equations given by

$$
R_{i j}-\frac{1}{2} R g_{i j}=8 \pi G t_{i j}
$$

where

$$
t_{i j}=T_{i j}^{(v)}+T_{i j}^{(m)}, \quad t_{; j}^{i j}=T_{; j}^{(v) i j}+T_{; j}^{(m) i j}=0 .
$$

Here $T_{i j}^{(m)}$ denotes the usual energy-momentum tensor of matter and $T_{i j}^{(v)}$ is the vacuum energy-momentum tensor, including $\Lambda_{0}$ and all other possible contributions to the vacuum energy density. The general form of $T_{i j}^{(v)}$ is not known but in particular it includes the so called variable $\Lambda$ models, where $T_{i j}^{(v)}=\frac{1}{8 \pi G} \Lambda(t) g_{i j}$, for some smooth scalar function of time $\Lambda(t)$. In this case the general conservation law in (1) becomes simply [2]

$$
\dot{\Lambda}=-g_{0 i} T_{; j}^{(m) i j}
$$

To complete our set of equations, we add a state equation generically expressed as

$$
p=(\gamma-1) \rho,
$$

In periods of time when matter becomes almost absolutely conserved we obtain $T^{(m) i j}{ }_{; j} \approx 0$ and $\Lambda(t) \approx$ constant, which could in principle be adjusted to match the observed cosmological constant.

In spite of many efforts, it appears to be difficult if not impossible to find a scalar field $\Lambda(t)$ satisfying the appropriate Klein-Gordon's equation in curved space- 
time with the total energy momentum tensor as a source [1]. This suggets that the vacuum could be a field of different nature [3]. For example, from (11) we see that $\Lambda(t)$ should behave just like the scalar curvature $R$ [4]. Nonetheless, we cannot use any linear combination of $R$ as $\Lambda(t)$, simply because $R$ is already included in left hand side of (11).

In another example $\Lambda(t)$ is taken to be a function of the space-time metric, so that its dynamics follows from the generalized gravitational field equations (1). Along this line some interesting models have been proposed [5], [6]. Nonetheless, each of these models use its own ad hoc ansatz for $\Lambda(t)$ and we still lack a proper explanation for this function, or more generally for the vacuum tensor $T_{i j}^{(v)}$.

Assuming that the vacuum is of geometrical nature, then it should reflect some geometrical property of the space-time which is not included in the left hand side of (11). For example, $\Lambda(t)$ could be made of a higher order contraction of curvature tensors, or even from some other manifestation of the space-time geometry, such as the extrinsic curvature. In this case, the observed small value of $\Lambda_{e f f} / 8 \pi G$ would be a symptom of the weak bending of the universe with respect to its tangent plane.

Based on the fact that the scalar curvature of the space-time may be decomposed into extrinsic components, we explore the possibility that the vacuum energy momentum tensor is related to the extrinsic curvature. In the next section we express the vacuum tensor $T_{i j}^{(v)}$ in terms of the extrinsic curvature of the space-time. Section 3 describes several extensions of the FRW model adapted to the more general equations (10). Finally, using a perturbative analysis we show in section four that the extrinsic curvature behaves as a spin-2 field, generally independent of the metric.

\section{Embedded Cosmologies}

If a given manifold is bent but not stretched, its intrinsic curvature does not change but its extrinsic curvature, which measures the deviation from the tangent plane, would change. Thus, two distinct cosmological models with the same intrinsic curvatures could exibit different bendings. We may ask if that difference would have 
any intrinsically observable effect. We will see that for the same matter distribution, the difference between the two extrinsic curvatures corresponds to different vacuum configurations. This means that the vacuum tensor can be written explicitly in terms of that extrinsic curvature (sometimes refered to as the second fundamental form).

Consequently, the observed $\Lambda(t)$ can be taken as an experimental evidence of the bending of the universe. Reciprocally, by specifying the dynamics of the extrinsic curvature we would be able to describe a number of interesting scenarios where $\lambda(t)$ rolls down to zero, remaining in that condition untill today..

The existence of this extrinsic curvature is a consequence of the well know mathematical property that any manifold, including space-times, can always be regarded as a subspace of a 5-dimensional space. Therefore, such subspace structure is not only compatible with, but also complementary to the exclusively metric description of space-time.

To understand the nature of the extrinsic curvature, we introduce a set of coordinates $X^{\mu}$, in a non flat 5 -dimensional manifold $\mathcal{M}_{5}$, such that

$$
g_{i j}=X_{, i}^{\mu} X_{, j}^{\nu} \mathcal{G}_{\mu \nu}, \quad N^{\mu} X_{, i}^{\nu} \mathcal{G}_{\mu \nu}=0, \quad \epsilon= \pm 1=N^{\mu} N^{\nu} \mathcal{G}_{\mu \nu}
$$

Here $N^{\nu}$ are the components of a vector field $N$ orthogonal to the space-time and $\mathcal{G}_{\mu \nu}$ are the components of the metric of $\mathcal{M}_{5}$. The necessary and sufficient conditions for the existence of a solution $X^{\mu}$ of (5), is that there is a tensor $b_{i j}$ satisfying the Gauss-Codazzi equations:

$$
\begin{aligned}
R_{i j k l} & =2 \epsilon b_{i[k} b_{l] j}+\mathcal{R}_{i j k l}, \\
\nabla_{[k} b_{i] j} & =\mathcal{R}_{i j k 5}
\end{aligned}
$$

where $\mathcal{R}_{i j k l}$ and $\mathcal{R}_{i j k 5}$ represent projections of the Riemann tensor of $\mathcal{M}_{5}$ along the directions $X_{, i}^{\mu}$ and $N^{\mu}$ respectivelly. The covariant derivative $\nabla_{i}$ is calculated with respect to $g_{i j}$. In terms of $X^{\mu}$, the tensor $b_{i j}$ may be expressed as

$$
b_{i j}=-X_{, i}^{\mu} N_{, j}^{\nu} \mathcal{G}_{\mu \nu}
$$

\footnotetext{
${ }^{1}$ Most known embedding spaces $\mathcal{M}_{D}$ are flat with $D \leq 10$. Here for generality we assume a curved space with $D=5$, so that a large class of comological models can be accomodated. Our conventions are: Lower case Latin indices run from 0 to 3 . All Greek indices run from 1 to 5 . $R_{i j}=g^{m n} R_{m i j n}$. The space-time signature is $(+++-)$.
} 
Contraction of (6) gives an important equation:

$$
R_{i j}-\frac{1}{2} R g_{i j}=t_{i j}(b)+\frac{4}{5}\left(\mathcal{R}_{i j}-\frac{2}{5} \mathcal{R} g_{i j}\right),
$$

where $\mathcal{R}_{i j}$ and $\mathcal{R}$ are the Ricci tensor and scalar curvatures of $\mathcal{M}_{5}$ respectively, and

$$
t_{i j}(b)=\epsilon\left(b_{i m} b_{j}{ }^{m}-h b_{i j}-\frac{1}{2}\left(\kappa^{2}-h^{2}\right) g_{i j}\right)
$$

where

$$
\kappa^{2}=b_{m i} b^{m i}, \quad h^{2}=\left(g^{i j} b_{i j}\right)^{2}
$$

are the extrinsic scalar and the mean curvatures respectively. Comparing with (1) we obtain

$$
T_{i j}^{(v)}=\frac{t_{i j}(b)}{8 \pi G}+\frac{1}{8 \pi G} \frac{4}{5}\left(\mathcal{R}_{i j}-\frac{2}{5} \mathcal{R} g_{i j}\right)-T_{i j}^{(m)}
$$

and the cosmological function in terms of $b_{i j}$ is defined by

$$
\Lambda(t) \stackrel{\text { def }}{=} 2 \pi G \operatorname{tr}\left(T_{i j}^{(v)}\right)=\frac{\epsilon}{4}\left(h^{2}-\kappa^{2}\right)-\frac{4}{25} \mathcal{R}-2 \pi G T_{i}^{(m) i} .
$$

From the contracted Bianchi identity, it follows that the generalized conservation rule in (11) is equivalent to the identity

$$
t^{i j}(b)_{; j}=0
$$

The dynamics of the field $b_{i j}$ is constrained by the Codazzi equation (7) which has no corresponding in the pure Riemannian geometry. Equations (9) say that the space-time curvature results from the blend of matter and vacuum energy densities, represented by $t_{i j}(b)$.

\section{FRW Example}

As an illustration, consider the Friedmann-Robertson-Walker (FRW) parametrization, with coordinates $\left(x^{1}, x^{2}, x^{3}, x^{4}\right)=(r, \theta, \phi, t)$ :

$$
d s^{2}=-d t^{2}+a^{2}(t)\left[d r^{2}+f^{2}(r)\left(d \theta^{2}+\sin ^{2} \theta d \phi^{2}\right)\right]
$$


with $f(r)=r, \sin r, \sinh r$ corresponding to $k=0,+1,-1$ (spatially flat, closed, open respectively). We use the energy-momentum tensor:

$$
T^{(m) i j}=p g^{i j}+(p+\rho) U^{i} U^{j}, \quad g_{i j} U^{i} U^{j}=-1 .
$$

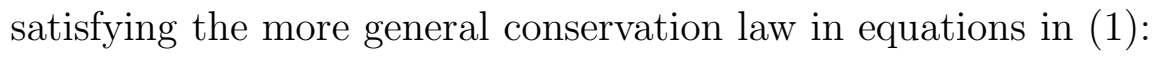

$$
\dot{\Lambda}(t)=8 \pi G(\dot{\rho}+3 H \gamma \rho)
$$

This cosmological model can be embedded in a 5-dimensional flat space with metric signature $(++++-)$ so that $g_{55}=\epsilon=+1$ [7]. It follows that equations ( 6.,7), reduce to

$$
\begin{aligned}
R_{i j k l} & =2 b_{i[k} b_{l] j}, \\
b_{k[i ; j]} & =0 .
\end{aligned}
$$

and the contracted form of (15) is

$$
R_{i j}-\frac{1}{2} R g_{i j}=t_{i j}(b)
$$

Instead of calculating $b_{i j}$ from (8), we may solve (16) directly obtaining

$$
b_{11}=b(t), b_{22}=f^{2}(r) b(t), b_{33}=(f(r) \sin \theta)^{2} b(t), b_{00}=-\frac{1}{\dot{a}} \frac{d}{d t}\left(\frac{b}{a}\right),
$$

where $b(t)$ is an arbitrary function of $t$ and all other components are zero. Replacing these values in (17), we obtain

$$
\begin{aligned}
\frac{\ddot{a}}{a} & =\frac{b}{a^{2} \dot{a}} \frac{d}{d t}\left(\frac{b}{a}\right), \\
a \ddot{a}+2 \dot{a}^{2}+2 k & =\frac{b}{a^{2} \dot{a}} \frac{d}{d t}(a b) .
\end{aligned}
$$

While the left hand sides of these equations are the familiar FRW expressions, the right hand sides, built with $b_{i j}$ are new. Eliminating $\ddot{a}$ among those equations, we obtain a single relation between $b$ and $a$ :

$$
\dot{a}^{2}+k=\frac{b^{2}}{a^{2}} .
$$


Denoting the Hubble parameter $H=\frac{\dot{a}}{a}$ and the relative change of the extrinsic curvature by $B=\frac{\dot{b}}{b}$, we may write

$$
b_{a b}=\frac{b}{a^{2}} g_{a b}, \quad b_{00}=\frac{b}{a^{2}}\left(\frac{B}{H}-1\right) g_{00}, \quad a, b=1 . .3
$$

The extrinsic scalar and mean curvatures are respectively

$$
\kappa^{2}=\frac{b^{2}}{a^{4}}\left(\frac{B^{2}}{H^{2}}-2 \frac{B}{H}+4\right) \quad h=\frac{b}{a^{2}}\left(\frac{B}{H}+2\right)
$$

and the vacuum energy-momentum tensor in terms of $b(t)$ is given by

$$
\begin{aligned}
T_{a b}^{(v)} & =\frac{1}{8 \pi G} \frac{b^{2}}{a^{4}}\left(\frac{2 B}{H}-1\right) g_{a b}-p g_{a b}, \\
T_{00}^{(v)} & =-\frac{1}{8 \pi G} \frac{3 b^{2}}{a^{4}}-\rho .
\end{aligned}
$$

The cosmological function (12) expressed in terms of the extrinsic geometry is

$$
\Lambda(t)=\frac{6 b^{2}}{4 a^{4}} \frac{B}{H}-2 \pi G(3 p-\rho) .
$$

Let us examine the meaning of the extrinsic curvature in some particular situations. Defining a new function $\omega(t)$ by $b=e^{\omega} a^{2}$, it follows that

$$
B=2 H+\dot{\omega}
$$

and (21) becomes $\dot{a}^{2}+k=e^{2 \omega} a^{2}$. Comparing with the extended Friedmann equation (with $\Lambda(t)$ )

$$
\dot{a}+k=\frac{8 \pi G}{3}\left(\rho-\frac{\Lambda(t)}{8 \pi G}\right) a^{2},
$$

we obtain $e^{2 \omega}=\frac{8 \pi G}{3} \rho_{T}$, where $\rho_{T}=\rho-\Lambda(t) / 8 \pi G$ is the total energy density. The derivative of this equation gives

$$
\frac{1}{2} \frac{\dot{\rho}_{T}}{\rho_{T}}=\dot{\omega}=B-2 H
$$

This can be integrated producing the general expression for the matter energy density in terms of $B, H$ and $\Lambda(t)$ :

$$
\rho_{T}=\rho_{0 T} e^{\int 2(B-2 H) d t}
$$


where $\rho_{0 T}$ is an integration constant. In particular, when $\omega=\omega_{0}=$ constant, that is when the extrinsic curvature is such that $B=2 H$, then $\rho$ and $\Lambda(t)$ become constants. It also follows from (22) that in this case $b_{i j}$ is conformally related to the metric:

$$
b_{i j}=e^{\omega_{0}} g_{i j}
$$

Replacing $b_{i j}$ in (15), we obtain

$$
R_{i j k l}=2 e^{2 \omega_{0}} g_{i[k} g_{l] j}
$$

showing that the space-time has constant curvature, which means in our example a deSitter universe. Let us suppose now that $\omega$ starts fluctuating. For example, we may have a membrane model of universe characterized by the condition $h=0$ [9]. From (23), this implies that $B=-2 H$, corresponding to $\dot{\omega}<0$. Integrating this relation we obtain $b=\frac{b_{0}}{a^{2}}$ and replacing in (26), we obtain a total density decaying as $\frac{1}{a^{8}}$, which means a universe filled with some kind of hyper-stiff combination of vacuum and matter [8], decaying to zero at a later stage. From (12) we see also that the sign of $\Lambda(t)$ is governed by the mean curvature of the space-time and the signature of $\mathcal{M}_{D}$. In a membrane universe with $h=0$ and $\epsilon=1$, we would have $\Lambda(t)<0$ [9]. On the other hand, when $\dot{\omega}>0$ or, equivalently when $B>2 H$, we have a universe where $\Lambda(t)$ and $\rho(t)$ would increase as positive powers of $a(t)$, leading to somewhat strange universes without the primordial singularity.

So far we have used only a general theorem on submanifolds to cosmology, without any further assumptions. Let us now consider a condition on the extrinsic curvature expressed as

$$
B=\alpha H
$$

where $b_{0}$ is an integration constant and where $\alpha$ is some yet unknown parameter. Since $B$ is a measure of the relative variation of the normal $N$, and $H$ is always decreasing with time, (27) has the reasonable interpretation that the relative variation of the normal vector $N$ decreases in proportion to the relative expansion of the universe. From (27) we have that $b=b_{0} a^{\alpha}$ so that $\Lambda(t)$ can be written as

$$
\Lambda(t)=\Lambda_{0} \alpha a^{2(\alpha-2)}-2 \pi G(3 p-\rho)
$$


where $\Lambda_{0}$ is a constant. The total density becomes

$$
\rho_{T}=\rho_{T} a^{2(\alpha-2)}
$$

When $\alpha=2$, we have a constant curvature space with a constant density. We may fix this constant asuming that it corresponds to the total critical density: $\rho=\rho_{T c r}$. In this case, the relative total density of vacuum and matter is

$$
\Omega_{T}=\frac{\rho_{T}}{\rho_{T c r}}=a^{2(\alpha-2)}
$$

As we see, depending on the value of $\alpha$ we obtain different scenarios, not necessarily corresponding to realistic models. The following table shows the decay $h, \Lambda$ and $\Omega_{T}$ corresponding to some values of $\alpha$, where we have set $\Lambda_{0}=1$ and used $3 p=\rho$ :

\begin{tabular}{|c|c|c||c|c|c||c|c|}
\hline & $\begin{array}{c}\text { mem- } \\
\text { branes }\end{array}$ & $\begin{array}{c}\text { stiff vac } \\
+ \text { matter }\end{array}$ & $\begin{array}{c}\text { radia-. } \\
\text { tion }\end{array}$ & $\begin{array}{c}\text { vac+matt. } \\
\text { dust }\end{array}$ & $\begin{array}{c}\text { deSitter } \\
\text { inflation }\end{array}$ & $\begin{array}{c}\text { strange } \\
\text { worlds }\end{array}$ & $\begin{array}{c}\text { stranger } \\
\text { worlds }\end{array}$ \\
\hline$\alpha=$ & -2 & -1 & 0 & 1 & 2 & 3 & 4 \\
\hline$h \leadsto$ & 0 & $1 / a^{3}$ & $2 / a^{2}$ & $3 / a$ & 4 & $5 a$ & $6 a^{2}$ \\
\hline$\Lambda \leadsto$ & $-2 / a^{8}$ & $-1 / a^{6}$ & 0 & $1 / a^{2}$ & 2 & $3 a^{2}$ & $4 a^{4}$ \\
\hline$\Omega_{T} \leadsto$ & $1 / a^{8}$ & $1 / a^{6}$ & $1 / a^{4}$ & $1 / a^{2}$ & 1 & $a^{2}$ & $a^{4}$ \\
\hline
\end{tabular}

table1: some values of $\alpha$ and corresponding scenarios

We can now replace $b(t)$ in (21) to obtain the expansion equation ( $\alpha=$ constant)

$$
\dot{a}^{2}+\kappa=b_{0}^{2} a^{2(\alpha-1)}
$$

It is quite conceivable that the proportion between $B$ and $H$ vary in different periods of the history of the universe. As table 1 suggests, $\alpha$ could be a function of time. One possible sequence would be that $\alpha \gtrsim 2$ at the beginning of the universe, with a density incresing at the rate of $a^{2}$, followed by a deSitter inflation with $\alpha=2, \Lambda=$ constant and $\Omega_{T}=1$. Next we would have a post inflationary period with $\alpha \lesssim 1$ when $\Lambda \leadsto 1 / a^{2}$. The other remaining values of $\alpha$ may not correspond to physically interesting models.

The following graphs display $\Lambda(t)$ and $\Omega(t)$ as a functions of the expansion factor $a(t)$ and of a possible continuous parameter $\alpha$. As we see in Fig.1, the strip between

\footnotetext{
${ }^{2}$ The $a$ scale has been roughly divided in 3 parts only: the early, middle and late universe. For plotting compatibility we have eliminated the singularity at $a=0$ [10.
} 
$\alpha \approx 0$ and the inflation line $\alpha \approx 2$ corresponds to the most common scenarios. In this region, $\Lambda(t)$ starts as a positive value but fall rapidly to zero during the early stages of the universe, remaining in that condition untill today. The values $\alpha>2$ represent a region where $\Lambda(t)$ has a polynomial increases at late universe. On the other hand, the regions located at $\alpha<0$ have an opposite result, where $\Lambda(t)$ starts with negative values but rapidly increases to zero at the early stages of the universe.

Fig.1: $\Lambda$ as continuous functions of $\alpha$ and $a$

Figure 2 shows the behavior of the relative total density $\Omega_{T}$ as a function of $a(t)$ and $\alpha$. Again, not all values of $\alpha$ corresponds to realistic models. From (27) we see that for the deSitter inflation line at $\alpha=2$ we have $\Omega_{T}=1$, suggesting that at the early universe $\alpha$ could have been slightly larger than 2. If we assume that the observed density is the result of the contribution of the vacuum and matter, that is, $\rho_{T}$, then we may take that $0.1 \leq \Omega_{T} \leq 2$. Consequently, today's universe would be somewhere in the region defined by $1<\alpha<2$, which in accordance with Fig.1 predicts $\Lambda(t) \approx 0$ at late universe. The non integer values of $\alpha$ in this domain seem to fit in a large classe of acceptable models. 
Fig.2: $\Omega_{T}$ as continuous functions of $\alpha$ and $a$

\section{Spin-2 Vacuum}

The hypothesis (27) should be replaced by a dynamical equation for the extrinsic curvature.

The obvious candidate for the Lagrangia would be constructed with the second invariant of the extrinsic curvature $K=\epsilon\left(\kappa^{2}-h^{2}\right)$, so that $K \sqrt{-g}$ is the extrinsic version of the Einstein-Hilbert Lagrangian. As it happens, the Euler-Lagrange equations derived from this expression with respect to $g_{i j}$ does not reproduce $t_{i j}(b)$. This can be explained by the fact that $K$ is homogeneous of degree 2 in $g_{i j}$, while EinsteinHilbert's Lagrangian is homogeneous of degree -1, leading to an inconsistency. To obtain the correct Lagrangian we notice that

$$
\frac{\partial}{\partial g^{i j}}(\sqrt{K} \sqrt{-g})=\frac{1}{\sqrt{K}} t_{i j}(b) \sqrt{-g}
$$

Therefore a Lagrangian which does not depend on derivatives of $g_{i j}$ would be given 
by the classical path integral over all embedded geometries:

$$
\mathcal{L}_{1}=\int \sqrt{K} \frac{\partial}{\partial g^{i j}}(\sqrt{K} \sqrt{-g}) d g_{i j},
$$

We can easily see that the Euler-Lagrange equations for $R \sqrt{-g}-\mathcal{L}_{1}$ with respect to $g_{i j}$ reproduce equation (17). On the other hand, the Euler-Lagrange equations with respect to $b_{i j}$ gives only an algebraic, expression (that is, not involving derivatives) on this field, so that $\mathcal{L}_{1}$ is not telling the whole story. To write the complete Lagrangian we need a deeper insight on the nature of the field $b_{i j}$, which can be obtained from a perturbative analysis addapted to the embedded cosmological models.

A space-time perturbation may be intuitively conceived as a local growth or deformation of a given background geometry. Mathematically speaking, this deformation may be described by a shift of the background along some transverse (that is, not tangent) vector field $\zeta$, producing a one parameter family of manifolds. The perturbed or physical space-time is a manifold with the same differentiable structure as the background, whose points are identified with all points along the integral curve of $\zeta$. Therefore, the metric of the perturbed manifold depends on the family parameter and it may be calculated by the Lie transport of the background metric along the integral curve [11. Thus, different perturbations are generated by different choices of transverse vectors $\zeta$. Since this is a transverse vector, its tangent component induce a coordinate transformations, producing a "coordinate gauge" condition on the perturbation. Of course, this is undesirable and should be filtered out. Only the perturbations which are independent of coordinate gauges are physically meaningful [12] and correspond to a density perturbation [13, [14]. Although this perturbation is described as a classical process, it eventually started from the backreactions of the fluctuations of quantum fields interacting with the classical gravitational field of the background. Here we merelly apply the above definition to the case of a space-time perturbation given by the tensor $b_{i j}$.

A family of embedded submanifolds of the flat space $\mathcal{M}_{5}$, in the neighborhood of that space-time, may be described by the Cartesian coordinates

$$
Z^{\mu}=X^{\mu}+s N^{\mu},
$$


where $s$ is a parameter such that $s=0$ correspond to the original background spacetime. In this case, a transverse vector can be written as

$$
\zeta^{\mu}=c^{i} X_{, i}^{\mu}+c N^{\mu}, \quad c \neq 0 .
$$

If $Q$ is a geometrical object defined in the background, then the corresponding change of $Q$ produce by the above perturbation is given by the Lie derivative $£_{\zeta} Q$. For a small value of the parameter $s$, the linear or first order perturbation is

$$
\stackrel{(1)}{Q}=Q+s £_{\zeta} Q .
$$

Consider two distinct linear perturbations of the same object $Q$ generated by two transverse vectors $\zeta$ and $\zeta^{\prime}$. Then,

$$
\stackrel{(1)}{Q^{\prime}}-\stackrel{(1)}{Q}=£_{\xi} Q-£_{\eta} Q
$$

where $\xi=\left(s^{\prime} c^{i}-s c^{i}\right) X_{, i}^{\mu}$ is a tangent vector and $\eta=\left(s^{\prime} c^{\prime}-s c\right) N^{\mu}$ is a normal vector. Suppose $\eta$ could be set to zero, without any further conditions imposed on $\xi$, which remains arbitraryf. In this case, the two perturbations would be equal whenever $£_{\xi} Q=0$ for any $\xi$. This implies that we could eliminate the perturbation by an infinitesimal coordinate transformation. Such condition can be satisfied only by a very special class of geometric objects. The metric could never be one such object unless all vector fields of the space-time are Killing vector fields. One possible solution to the above coordinate gauge problem is to take the normal vector $N$ as the transverse vector (with a non zero signature). In this way, no coordinate transformation can interfere with the perturbation. Next, to construct the perturbation of an object, we first determine the perturbation of a tetrad field $\left\{h_{j}^{i}\right\}$ and then contract the object with the perturbed tetrad [15]. The linear perturbation of $\left\{h_{j}^{i}\right\}$ generated by $N$ is

$$
h_{j}^{i}=h_{j}^{i}+s £_{N} h_{j}^{i}
$$

\footnotetext{
${ }^{3}$ This would be the case if the signature of $N$ is taken to be zero as in [11, where there is no measure for the length $c$.
} 
and the physical components of the metric in the perturbed tetrad is given by

$$
\stackrel{(2)}{g_{i j}}=\stackrel{(1)}{h_{i}^{m}} \stackrel{(1)}{h_{j}^{n}} g_{m n}
$$

Since our perturbed space-times have a metric induced by $\mathcal{G}_{\mu \nu}$ (or $\eta_{\mu \nu}$ in the flat case), we may replace the tetrad $\left\{h_{j}^{i}\right\}$ by a vielbein $\left\{l_{i}^{\mu}\right\}$ and the perturbed metric will be given by

$$
\stackrel{(2)}{g_{i j}}=\stackrel{(1)}{l_{i}^{\mu}} \stackrel{(1)}{l_{j}^{\nu}} \mathcal{G}_{\mu \nu}
$$

Let us consider the unperturbed vielbein to be $l_{i}^{\mu}=X_{, i}^{\mu}$ and, just for the sake of intuition, that the normal $N$ points towards the most convex side of the space-time. Then, the perturbed vielbein along this normal is

$$
\stackrel{(1)}{l_{i}^{\mu}}=X_{, i}^{\mu}-s £_{N} X_{, i}^{\mu}=X_{, i}^{\mu}+s N_{, i}^{\mu}
$$

so that the second order geometric perturbation of the metric is

$$
\stackrel{(2)}{g}_{i j}(x, s)=\stackrel{(1)}{l}_{i}^{\mu} \stackrel{(1)}{l}_{j}^{\nu} \eta_{\mu \nu}=X_{, i}^{\mu} X_{, j}^{\nu} \eta_{\mu \nu}+2 s N_{, i}^{\mu} X_{, j}^{\nu} \eta_{\mu \nu}+s^{2} N_{, i}^{\mu} N_{, j}^{\nu} \eta_{\mu \nu}
$$

or, using (8),

$$
\stackrel{(2)}{g}_{i j}=g_{i j}-2 s b_{i j}+s^{2} g^{m n} b_{i m} b_{j n}=g^{m n}\left(g_{i m}-s b_{i m}\right)\left(g_{j n}-s b_{j n}\right) .
$$

An approximate field equation for $b_{i j}$ can be obtained from the linear perturbation of the metric (assuming $s^{2}<<s$ in (34)):

$$
\stackrel{(1)}{g_{i j}} \approx g_{i j}-2 s b_{i j}
$$

Replacing this in (1), and applying the usual deDonder gauge $\left(b_{j}^{i}-h / 2 \delta_{j}^{i}\right)_{, i}=0$, we obtain

$$
\square^{2}\left(b_{i j}-\frac{1}{2} h g_{i j}\right)-R_{i}{ }^{k l}{ }_{j}\left(b_{k l}-\frac{1}{2} h g_{k l}\right)=8 \pi G\left(T_{i j}^{(v)}+T_{i j}^{(m)}\right),
$$

so that the extrinsic curvature is a spin-2 field over the background with the total energy-momentum tensor as source. Now, if a spin-2 field has the total energy momentum tensor as a source, it must necessarily be derived from a Einstein-Hilbert 
type Lagrangian $\mathcal{L}_{2}=R\left(b_{i j}\right) \sqrt{-\operatorname{det}\left(b_{i j}\right)}$, where $b_{i j}$ takes the place of the metric in all expressions and contractions [16]. The resulting Lagrangian would therefore be

$$
\mathcal{L}_{b}=\mathcal{L}_{1}+\mathcal{L}_{2}=\int \sqrt{K} \frac{\partial}{\partial g^{i j}}(\sqrt{K} \sqrt{-g}) d g_{i j}+R\left(b_{i j}\right) \sqrt{\frac{\operatorname{det}\left(b_{i j}\right)}{g}} \sqrt{-g}
$$

Notice that the dependence on $g_{i j}$ in the second part of this Lagrangian is only fictitious. We may now set the total Lagrangian as

$$
\mathcal{L}=R(g) \sqrt{-g}-\mathcal{L}_{b}
$$

This Lagrangian, tells us how the universe bends in response to the vaccum energy and in particular determines the behavior of $\Lambda(t)$. The corresponding field equations with respect to $g_{i j}$ are

$$
R_{i j}(g)-\frac{1}{2} R(g) g_{i j}=t_{i j}(b)=8 \pi G\left(T_{i j}^{(v)}+T_{i j}^{(m)}\right)
$$

while the field equations with respec to $b_{i j}$ are

$$
R_{i j}(b)-\frac{1}{2} R(b) b_{i j}=f_{i j}(b)
$$

where $f_{i j}(b)$ is defined by

$$
\frac{\partial f_{i j}(b)}{\partial g^{m n}}=\epsilon\left[g_{(i m} b_{n) j}-\left(b_{i j}-h g_{i j}\right) g_{m n}-\left(g_{i j} b_{m n}-h g_{i m} g_{j n}\right)\right] \sqrt{-g} .
$$

This tensor is not a source term for $b_{i j}$ and is not zero in general. Further constraints may be imposed on the extrinsic geometry of the space-time by assigning specific values for $f_{i j}$.

In summary, the vacuum energy was expressed in terms of the extrinsic curvature of the space-time. In a first exploratory analysis we have used the geometric constraint $B=\alpha H$, obtaining several possible scenarios where $\Lambda(t)$ decays rapidly to zero and $\Omega$ becomes 1 or a value smaller than 1 in its late stages. Next, a dynamic condition on the extrinsic curvature was defined by a perturbation of the space-time generated by $b_{i j}$. We concluded that this field behaves as a spin-2 field over the background, 
with the total energy-momentum tensor as its source. Once this was understood, we applied a general theorem due to S. Gupta to derive the finite Lagrangian for $b_{i j}$. In a subsequent paper, we will apply those results to examine the density perturbation induced by the extrinsic curvature in the FRW example.

Acknowledgements:

The authors wish to thank the warm hospitality received at Fermilab. This work was supported in part by grants from CAPES, CNPq, DOE and NASA grant \# NAGW-2381. 


\section{References}

[1] S. Weinberg, Rev. Mod. Phys. $\underline{\mathbf{6 1}}, \mathbf{1},(\mathbf{1 9 8 9})$

[2] M. Ozer \& M.O. Taha. Nucl. Phys $\underline{\mathbf{2 8 7}}, \mathbf{7 7 7},(\mathbf{1 9 8 7})$

[3] A. D. Dolgov. The Cosmological Constant Problem in The Quest for Fundamental Constants in Cosmology. J. Audazes \& J Tran Thanvan (eds). Editions Frontiers, Gif-Sur-Ivette, Cedex France, p. 227 (1989)

[4] S. M. Barr. Phys. rev D36, 1691, (1987)

[5] Wei Chen \& Yong-Shi Wu. Phys. Rev.D41, 695 (1990) .

[6] I. Waga. The Astrophys. Jour. 1ㄴ, 436 (1993)

[7] J. Rosen. Rev. Mod. Phys. 구, 204, (1965)

[8] B. K. Harrison, K.S. Thorne, M. Wakano and J. A. Wheeler. em Graviattion Theory and Gravitational Collapse. U. of Chicago Press, Chicago (1964)

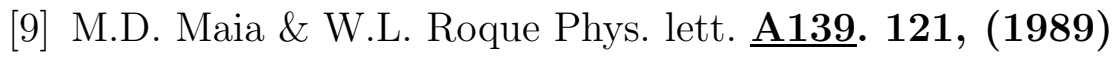

[10] S. Wolfram. Mathematica (2nd ed) Addison Wesley, N.Y (1991)

[11] R. Geroch. Commun. Math. Phys. $\underline{\mathbf{1 3}}, \mathbf{1 8 0 , ( 1 9 6 9 )}$

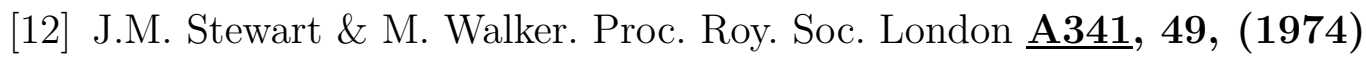

[13] J.M. Bardeen. Phys. Rev. D22, 1882, (1980)

[14] V. F. Mukanov, H.A. Feldman \& R.I. Brendenberg. Phys. Rep. 215,203 (1992)

[15] S. Chandresaker. The Mathematical Theory of Black Holes. Oxford University Press, Oxford (1992)

[16] S.N. Gupta. Phys. Rev $\underline{96}, 1683$ (1954) 
This figure "fig1-1.png" is available in "png" format from: http://arxiv.org/ps/gr-qc/9401005v1 
This figure "fig1-2.png" is available in "png" format from: http://arxiv.org/ps/gr-qc/9401005v1 